03

\title{
Электрическая площадь импульса при разделении и слиянии зарядов в вакууме
}

\author{
(C) Н.Н. Розанов \\ ${ }^{1}$ ФТИ им. А.Ф. Иофрфе, \\ 194021 Санкт-Петербург, Россия \\ ${ }^{2}$ Университет ИТМО, \\ 197101 Санкт-Петербург, Россия \\ e-mail: nnrosanov@mail.ru
}

Поступила в редакцию 13.12.2019 г.

В окончательной редакции 13.12.2019 г.

Принята к публикации 23.12.2019 г.

\begin{abstract}
Аналитически определено распределение электрической площади импульса, генерируемого зарядами, возникающей при ионизации исходно незаряженного микрообъекта, который после вызванной кулоновским притяжением зарядов рекомбинации вновь становится незаряженным. Показано, что при приближении к точкам, в которых мгновенная скорость частиц обращается в 0, электрическая площадь неограниченно растет по степенному закону (,правило $-3 / 2^{“}$ ).
\end{abstract}

Ключевые слова: униполярные импульсы излучения, электрическая площадь импульсов.

DOI: $10.21883 /$ OS.2020.04.49200.350-19

Квазиуниполярные импульсы электромагнитного излучения, которые обладают значительной постоянной составляющей, впервые обсуждались в работах [1-3] и экспериментально наблюдались в [4]. Их можно рассматривать как предельный случай уменьшения длительности импульсов - одной из наиболее актуальных проблем современной лазерной физики и техники [5]. Значимость таких импульсов подчеркивает то, что они в отличие от обычных биполярных импульсов оказывают однонаправленное и потому более эффективное воздействие на классические [6] и квантовые [7-11] микрообъекты. Как показано в [12], в волноводах с неодносвязным поперечным сечением возможна транспортировка квазиуниполярных импульсов на значительные расстояния.

Обзор различных способов генерации квазиуниполярных импульсов представлен в [13]. Существенным для реализации этих способов является правило сохранения электрической площади импульсов [14-16], определяемой соотношением $\mathbf{S}_{e}(\mathbf{r})=\int_{t=-\infty}^{+\infty} \mathbf{E}(\mathbf{r}, t) d t$ (здесь $\mathbf{r}$ и $t-$ пространственный радиус-вектор и время, а $\mathbf{E}-$ напряженность электрического поля). Примечательно, что в случае движущихся зарядов в вакууме из уравнений Максвелла следует замкнутая система уравнений для определения площади $\mathbf{S}_{E}$ через интегральную плотность зарядов $Q(\mathbf{r})=\int_{-\infty}^{+\infty} \rho(\mathbf{r}, t) d t$, где $\rho-$ плотность электрических зарядов [17]. Формально эта система совпадает с уравнениями электростатики [18], что позволяет находить электрическую площадь по (заданному) пространственному распределению интегральной плотности зарядов.

Пример такого подхода представлен в [19], где рассматривалось равноускоренное движение точечного за- ряда, вылетающего из электронной пушки и затем возвращающегося в нее под действием силы тяжести. Как показано в [19], при приближении к точке обращения в 0 мгновенной скорости заряда электрическая площадь неограниченно возрастает, что подтверждает возможность получения весьма больших значений этой величины. В то же время принятое в [19] пренебрежение полем электронной пушки после вылета из нее заряда может вызывать вопросы. Задачей настоящего сообщения служит решение родственной задачи, в которой исходно нейтральный микрообъект (например, атом) ионизуется и затем после возвращения вылетевшего электрона под действием кулоновского притяжения происходит рекомбинация. В этом случае не сложно учесть поля не только вылетевшего электрона, но и иона.

Напомним, что, согласно [17], для вакуума с зарядами из электродинамических уравнений Максвелла вытекает следующая замкнутая (при условии заданности распределения плотности зарядов) система уравнений для электрической площади:

$$
\operatorname{rot} \mathbf{S}_{E}=0, \quad \operatorname{div} \mathbf{S}_{E}=4 \pi Q .
$$

Решение этой системы, аналогичной уравнениям электростатики, находится с помощью введения „потенциала“ $\Phi_{S}$, подчиняющегося уравнению Пуассона

$$
\Delta \Phi_{S}=-4 \pi Q .
$$

Соответственно

$$
\Phi_{S}(\mathbf{r})=\int \frac{Q\left(\mathbf{r}^{\prime}\right)}{\left|\mathbf{r}-\mathbf{r}^{\prime}\right|} d \mathbf{r}^{\prime},
$$

после чего электрическая площадь определяется через градиент „потенциала“:

$$
\mathbf{S}_{E}=-\operatorname{grad} \Phi_{S}
$$


Применим указанный подход к решению простой задачи об ускоренном нерелятивистском движении зарядов. Пусть из исходно нейтрального микрообъекта (атома) спонтанно (радиоактивный распад ядра) или под действием внешнего ионизирующего излучения вырывается частица с зарядом $q_{1}$ и массой $m_{1}$ (например, электрон) с некоторой начальной скоростью. Оставшийся ион обладает зарядом $q_{2}=-q_{1}$ и массой $m_{2}$. В системе центра инерции скорости этих двух частиц $\mathbf{v}_{1}$ и $\mathbf{v}_{2}$ связаны соотношением $m_{1} \mathbf{v}_{1}+m_{2} \mathbf{v}_{2}=0$. Начальное расстояние между двумя зарядами естественно считать порядка размеров нейтрального микрообъекта. В дальнейшем между ними действует кулоновская сила притяжения. Поэтому сначала два заряда будут удаляться друг от друга, но затем, если их начальные скорости не слишком велики, удаление сменится приближением. Под действием кулоновского притяжения заряды сблизятся до исходного расстояния, при котором произойдет их рекомбинация. Естественно, что в рамках классической электродинамики мы не можем описать детально начальный и конечный этапы (ионизация и рекомбинация), но это не столь критично, поскольку основные выводы слабо зависят от величины начального (и конечного) расстояния между зарядами.

В такой постановке мы приходим к классической задаче двух тел, взаимодействующих через кулоновское притяжение, т.е. к кеплеровой задаче [20]. Вводя приведенную массу $m=m_{1} m_{2} /\left(m_{1}+m_{2}\right)$ и векторное расстояние между телами $\mathbf{r}_{12}=\mathbf{r}_{1}-\mathbf{r}_{2}$, выражаем через $\mathbf{r}_{12}$ индивидуальные положения частиц и их скорости:

$$
\begin{array}{ll}
\mathbf{r}_{1}=\frac{m_{2}}{m_{1}+m_{2}} \mathbf{r}_{12}, & \mathbf{r}_{2}=-\frac{m_{1}}{m_{1}+m_{2}} \mathbf{r}_{12}, \\
\mathbf{v}_{1}=\frac{m_{2}}{m_{1}+m_{2}} \mathbf{v}_{12}, & \mathbf{v}_{2}=-\frac{m_{1}}{m_{1}+m_{2}} \mathbf{v}_{12},
\end{array}
$$

При этом мы приходим к задаче о движении точечной частицы с массой $m$ в поле с потенциалом $U\left(r_{12}=-\left|q_{1} q_{2}\right| / r_{12}\right.$.

Хотя уравнение движения эффективной частицы интегрируется в квадратурах в общем виде, ограничимся более простым случаем, когда начальные скорости двух частиц обладают радиальным направлением. Тогда механический момент обращается в 0 и движение становится одномерным. Здесь нам достаточно воспользоваться законом сохранения механической энергии, т. е. сохранением величины

$$
E_{m}=\frac{1}{2} m v_{12}^{2}-\frac{\left|q_{1} q_{2}\right|}{r_{12}} .
$$

Энергия выражается через начальные данные при удалении $r=r_{0.12}$ и начальной скорости $v_{0.12}$ : $E_{m}=\frac{1}{2} m v_{0.12}^{2}-\frac{\left|q_{1} q_{2}\right|}{r_{0.12}}<0 \quad$ (последнее неравенство обеспечивает невозможность неограниченного удаления частиц друг от друга). Соответственно максимальное удаление, при котором скорость обращается в 0, $r_{\max , 12}=\left|q_{1} q_{2} / E_{m}\right|$. Само значение удаления меняется в пределах $0<r_{0.12}<r_{12}<r_{\max , 12}$. Текущее значение скорости зависит от $r_{12}$ следующим образом:

$$
v\left(r_{12}\right)= \pm V\left(r_{12}\right), \quad V\left(r_{12}\right)=\left(\frac{2}{m}\left|E_{m} \frac{r_{\max , 12}-r_{12}}{r_{12}}\right|\right)^{1 / 2} .
$$

Положение индивидуальных частиц и их скорости находятся по соотношениям (5).

Как и в [19], принимаем дельтаобразное распределение плотности зарядов

$$
\rho\left(\mathbf{r}_{\perp}, z, t\right)=\delta\left(\mathbf{r}_{\perp}\right) \sum_{i=1}^{2} q_{i} \delta\left(z-z_{i}(t)\right) .
$$

Здесь $\mathbf{r}_{\perp}=(x, y)$ - двумерный вектор поперечных координат, $z$ - продольная координата вдоль направления прямолинейного движения частиц, а $z_{i}-$ продольная координата $i$-й частицы. Ввиду аддитивности интегральной плотности заряда, „потенциала“ $\Phi_{S}$ и электрической площади их можно вычислить отдельно для каждой частицы и затем сложить. Соответственно

$$
Q_{i}\left(\mathbf{r}_{\perp, z}\right)=2 \int_{t_{0}}^{t_{\max }} \rho_{i}\left(\mathbf{r}_{\perp}, z, t\right) d t=\frac{2 q_{i}}{\left|\nu_{i}(z)\right|} \delta\left(\mathbf{r}_{\perp}\right),
$$

где

$$
\left|v_{i}(z)\right|=\left(\frac{2}{m_{i}}\left|E_{m}\right| \frac{\left|z_{\max , i}\right|-\left|z_{i}\right|}{\left|z_{i}\right|}\right)^{1 / 2} .
$$

Интегральная плотность заряда отлична от 0 только на траекториях частиц (на двух отрезках оси $z$, $\left.\left|z_{0, i}\right|<|z|<\left|z_{\max , i}\right|\right)$ и неограниченно возрастает при приближении к максимальным значениям удаления $\left|z_{\max , i}\right|$, где скорость частицы обращается в 0. Теперь „потенциал“ (3) для первой частицы принимает вид

$$
\begin{aligned}
\Phi_{1, S}\left(r_{\perp}, z\right) & =q_{1} \sqrt{\frac{2 m_{1}}{\left|E_{m}\right|}} \\
& \times \int_{z_{0,1}}^{z_{\max , 1}}\left\{\frac{z^{\prime}}{\left(z_{\max , 1}-z^{\prime}\right)\left[r_{\perp}^{2}+\left(z-z^{\prime}\right)^{2}\right]}\right\}^{1 / 2} d z^{\prime} .
\end{aligned}
$$

Правая часть (11) выражается в общем случае через эллиптические интегралы [21]. Ее вид упрощается на оси $z$ (при $r_{\perp}=0$ и $\left.z>z_{\max , 1}\right)$ :

$$
\begin{aligned}
& \Phi_{1, S}(0, z)=q_{1} \sqrt{\frac{2 m_{1}}{\left|E_{m}\right|}} \int_{z_{0,1}}^{z^{\max , 1}}\left\{\frac{z^{\prime}}{\left(z_{\max , 1}-z^{\prime}\right)}\right\}^{1 / 2} \frac{d z^{\prime}}{z-z^{\prime}} \\
& =q_{1} \sqrt{\frac{8 m_{1}}{\left|E_{m}\right|}}\left[(a-1) \frac{\pi}{2}-a \operatorname{arctg}\left(u_{0,1} / a\right)+\operatorname{arctg}\left(u_{0,1}\right)\right],
\end{aligned}
$$

где

$$
u_{0,1}^{2}=\frac{z_{0,1}}{\left(z_{\max , 1}-z_{0,1}\right)}, \quad a^{2}=\frac{z}{z-z_{\max , 1}} .
$$


При приближении к точке мгновенной остановки частицы $z \rightarrow z_{\max , 1}$ и при $z_{\max , 1} \gg z_{0,1}$ из $(12)$ и (13) следует

$$
\begin{aligned}
\Phi_{1, S}(0, z) & \approx \pi q_{1} \sqrt{\frac{2 m_{1} z_{\max , 1}}{\left|E_{m}\right|}}\left(z-z_{\max , 1}\right)^{-1 / 2}, \\
S_{E 1, z}(0, z) & =-d \Phi_{1, S} / d z \\
& \approx \pi q_{1} \sqrt{\frac{m_{1} z_{\max , 1}}{2\left|E_{m}\right|}}\left(z-z_{\max , 1}\right)^{-3 / 2},
\end{aligned}
$$

Примечательно, что в конечные выражения (14) не входит величина начального удаления зарядов $r_{0,12}$. Такая слабая чувствительность результата к величине этого модельного параметра вызвана тем, что максимальное удаление зарядов значительно превышает их первоначальное раздвижение. Аналогично выражение „потенциала“ и электрической площади и для второй частицы.

На оси движения частиц поперечные компоненты вектора электрической площади равны 0 , так что вблизи оси $z$-компонента доминирует. Таким образом, как и в рассмотренной в [19] задаче, при приближении к точке остановки заряженной частицы электрическая площадь неограниченно возрастает степенным образом по „правилу $-3 / 2^{\text {‘6. }}$.

При помещении микрообъекта, например атома, в окрестности точки остановки заряда на неподвижный микрообъект будет действовать импульс излучения с большой электрической площадью. Если же речь идет об ускорении заряженной частицы, то для эффективного ускорения необходимо максимизировать не локальную величину электрической площади, а ее обобщение для сопровождающей движущийся заряд системы координат (см. также [6]). Представляет интерес и решение обратной задачи потенциала с определением требуемого распределения „потенциала“ и плотности зарядов.

\section{Благодарности}

Автор благодарен М.В. Архипову за полезные обсуждения.

\section{Финансирование работы}

Исследование поддержано грантами РФФИ 19-0200312 и 20-32-70049, а также Программой президиума РАН „Математика и нелинейная динамика“.

\section{Конфликт интересов}

Автор заявляет, что у него нет конфликта интересов.

\section{Список литературы}

[1] Бессонов Е.Г. // ЖЭТФ. 1981. Т. 80. № 3. С. 852-858; Bessonov E.G. // Sov. Phys. JETP. 1981. V. 53. N 3. P. 433-436.
[2] Bessonov E.G. // Nucl. Instr. and Meth. A. 1991. V. 308. P. $135-139$.

[3] Бессонов Е.Г. // Квант. электрон. 1992. Т. 19. № 1. C. 35-39; Bessonov E.G.// Sov. J. Quant. Electron. 1992. V. 22. N 1. P. 27-31.

[4] Naumenko G., Shevelev M. // J. Instrumentation. 2018. V. 13. N 5. P. C05001.

[5] Электронный ресурс. Режим доступа: https://hightech.fm/2018/10/09/nobel-4.

[6] Розанов Н.Н., Высотина Н.В. // ЖЭТФ. Т. 157. В. 1. C. 63-67 (2020).

[7] Dimitrovski D., Solov'ev E.A., Briggs J.S. // Phys. Rev. Lett. 2004. V. 93. P. 083003.

[8] Dimitrovski D., Solov'ev E.A., Briggs J.S. // Phys. Rev. A. 2005. V. 72. P. 043411.

[9] Макаров Д.Н., Матвеев В.И. // Письма в ЖЭТФ. 2016. T. 103. B. 6. C. $464-468$.

[10] Розанов Н.Н. // Опт. и спектр. 2018. Т. 124. № 1. С. 75-77; Rosanov N.N. // Opt. Spectrosc. 2018. V. 124. N 1. P. 72-74.

[11] Arkhipov R.M., Arkhipov M.V., Babushkin I.V., Demircan A., Morgner U., Rosanov N.N. // Opt. Lett. 2019. V. 44. N 5. P. $1202-1205$.

[12] Розанов Н.Н. // Опт. и спектр. 2019. Т. 127. В. 6. C. 960-962; Rosanov N.N. // Opt. Spectrosc. 2019. V. 127. $\mathrm{N} 6$.

[13] Архипов Р.М., Архипов М.В., Шимко А.А., Пахомов А.В., Розанов Н.Н. // Письма в ЖЭТФ. 2019. Т. 109. № 10. C. 9-20; Arkhipov R.M., Arkhipov M.V., Shimko A.A., Pakhomov A.V., Rosanov N.N. // JETP Lett. 2019. V. 110. N 1. P. $15-24$.

[14] Розанов Н.Н. // Опт. и спектр. 2009. Т. 107. N 5. C.761-765; Rosanov N.N. // Opt. Spectrosc. 2009. V. 107. N 5. P. $721-725$.

[15] Rosanov N. N., Kozlov V.V., Wabnitz S. // Phys. Rev. A. 2010. V. 81. N 4. P. 043815

[16] Розанов Н.Н., Архипов Р.М., Архипов М.В. // УФН. 2018. T. 188. B. 12. C. 1347-1353; Rosanov N.N., Arkhipov R.M., Arkhipov M.V. // Physics-Uspekhi. 2018. V. 61. N 12. P. $1227-1233$.

[17] Розанов Н.Н.// Опт. и спектр. 2020. Т. 128. № 1. С. 95-97; Rosanov N.N.// Opt. Spectrosc. 2020. V. 128. N 1.

[18] Ландау Л.Д., Либиии, Е.М. Электродинамика сплошных сред. М.: Физматлит, 1982.620 с.

[19] Розанов Н.Н. // Письма в ЖТФ. 2020. Т. 46. № 4. С. 15-17; Rosanov N.N. // JETP Lett. 2020. V. 46. N 4.

[20] Ландау Л.Д., Либшии, Е.М. Механика. М.: Физматлит, 2012. 224 c.

[21] Градштейн И.С., Рыжик И.М. Таблицы интегралов, сумм, рядов и произведений. М.: Физматлит, 1962.1100 с. 